\title{
A Theory of Testing for Timed Automata
}

\author{
Frits Vaandrager \\ University of Nijmegen
}

\begin{abstract}
We present a generalization of the classic theory of testing for (finite state) Mealy machines to a setting of timed automata in the style of Alur and Dill.
\end{abstract}

